\title{
Isolation of a Ferroelectric Intermediate Phase in Antiferroelectric Dense
}

\section{Sodium Niobate Ceramics}

Hangfeng Zhang, ${ }^{1}$ Bin Yang, ${ }^{2}$ Haixue Yan $^{\star 3}$ and Isaac Abrahams ${ }^{* 1}$

${ }^{1}$ School of Biological and Chemical Sciences, Queen Mary University of London, Mile End Road London E1 4NS, U.K.

${ }^{2}$ Department of Electronic and Electrical Engineering, University of Chester, Chester, $\mathrm{CH} 2$ 4NU, U.K.

${ }^{3}$ School of Engineering and Materials Sciences, Queen Mary University of London, Mile End Road London, E1 4NS, U.K.

${ }^{*}$ Corresponding authors:

H. Yan: E-mail: h.x.yan@qmul.ac.uk $\quad$ Tel: $\quad+44(0) 2078825164$

I. Abrahams: E-mail: i.abrahams@qmul.ac.uk Tel: $\quad+44(0) 2078823235$

\section{Abstract}

Switchable ferroelectric/antiferroelectric ceramics are of significant interest for high power energy storage applications. Grain size control of this switching is an interesting approach to controlling polarization and hence dielectric properties. However, the use of this approach in technologically relevant ceramics is hindered by difficulty in fabricating dense ceramics with small grain sizes. Here an intermediate polar ferroelectric phase $\left(P 2_{1} m a\right)$ has been isolated in dense bulk sodium niobate ceramics by grain size control through spark plasma sintering methods. Our findings, supported by XRD, DSC, P-E (I-E) loops and dielectric characterization, provide evidence that the phase transition from the antiferroelectric (AFE) R-phase, in space group Pnmm, above $300^{\circ} \mathrm{C}$, to the AFE P-phase, in space group Pbma, at room temperature, always involves the polar intermediate $P 2{ }_{1}$ ma phase and that the $P 2{ }_{1} m a \rightarrow P b m a$ transition can be suppressed by reducing grain size.

\section{Graphical abstract}

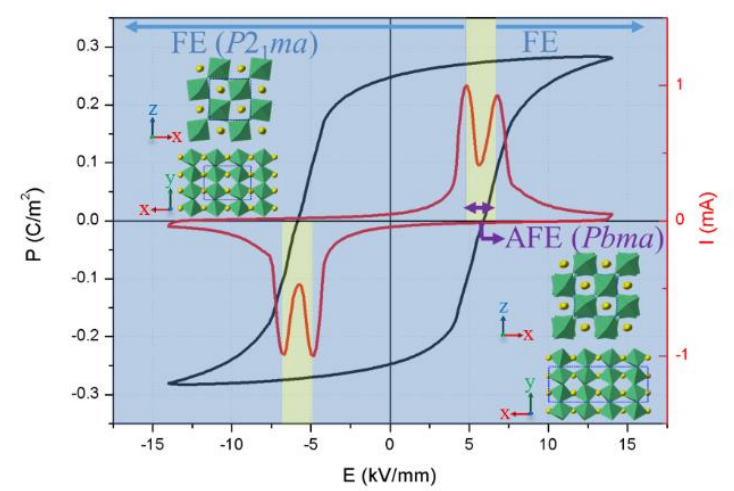

Keywords: Sodium niobate, ceramics, phase transitions, dielectric measurements, grain size. 


\section{Introduction}

The change in polarization that occurs on ferroelectric (FE)/antiferroelectric (AFE) switching in dielectric materials allows for important technical applications such as transducers, sensors, solid-state memories and capacitors [1-4]. The nature, scale and order of the associated phase transition is governed by many factors such as phonons, microstructure, defects and compositional homogeneity [5]. Of these, microstructure is particularly interesting and has been studied and manipulated in many systems such as $\mathrm{BaTiO}_{3}, \mathrm{~Pb}(\mathrm{Zr}, \mathrm{Ti}) \mathrm{O}_{3}$ and $\mathrm{Sr}_{2} \mathrm{Bi}_{4} \mathrm{Ti}_{5} \mathrm{O}_{18}$ [6-9]. For example, decreasing grain size in $\mathrm{BaTiO}_{3}$ leads to the suppression of tetragonal order in the crystal structure and the preservation of the paraelectric cubic phase to room temperature. The suppression of the cubic to tetragonal phase transition is attributed to the containment of single domains within single grains and the limiting of domain growth by the grain boundaries.

Sodium niobate $\left(\mathrm{NaNbO}_{3}\right)$ is an important lead-free AFE material and its derivatives such as calcium and strontium doped sodium niobate [10-13] and potassium substituted sodium niobate $\left(\mathrm{Na}_{1-x} \mathrm{~K}_{x} \mathrm{NbO}_{3}\right)$ [14-16], have shown great potential in high power energy storage and piezoelectric applications, respectively. $\mathrm{NaNbO}_{3}$ is particularly interesting, as it displays a rich variety of seven phases over the temperature range $173 \mathrm{~K}$ to $913 \mathrm{~K}$ [17]. $\mathrm{NaNbO}_{3}$ was originally identified by Barth, in 1925 , as a cubic perovskite $(a=3.906 \AA$ ), using X-ray powder diffraction [18]. Later work found the room temperature structure (P-phase) to be orthorhombically distorted in space group $P 222_{1}(a=5.504 \AA, b=5.568 \AA, c=15.52 \AA$ ) [19], while variable temperature studies suggested a monoclinic distortion at room temperature in space group $P 2 / m\left(a=3.9114 \AA, b=3.8813 \AA, c=3.9114 \AA, \beta=90.67^{\circ}\right)$ [20]. The structure first presented in 1969 by Sakowski-Cowley et al. [21] in space group Pbma ( $a=5.506$ $\AA, b=5.566 \AA, c=15.52 \AA$ ) is now accepted as correctly describing the room temperature structure. Evidence, from high-resolution powder neutron diffraction, suggested a small monoclinic distortion away from true orthorhombic symmetry $(a=$ $5.5101 \AA, b=5.5717 \AA, c=15.5181 \AA, \gamma=89.94^{\circ}$ ) [22,23], but it was later found that this distortion was associated with a room temperature polar phase approximating to orthorhombic symmetry in space group $P 2{ }_{1} m a(a=5.571 \AA$, $b=7.766 \AA, c=5.513 \AA$ ), which can coexist with the non-polar phase (Pbma) [24]. First-principles calculations confirm similar free energies for the polar $\left(P 2{ }_{1} m a\right)$ and non-polar $(P b m a)$ phases in $\mathrm{NaNbO}_{3}$ [25]. Cross and Nicholson found a double polarization-electric field (P-E) hysteresis loop in a $\mathrm{NaNbO}_{3}$ single crystal at a field of ca. $10 \mathrm{kV} \mathrm{mm}^{-1}$ and proposed a field induced transition from antiferroelectric (non-polar) to a ferroelectric (polar) phase 
[26]. This was confirmed by Shuvaeva et al. who showed by single crystal X-ray diffraction a field induced change from Pbma (AFE) to $P 2{ }_{1}$ ma (FE) symmetry [27]. Under high electric field, Ulinzheyev et al. found another field induced phase transition from orthorhombic to tetragonal through measurements of birefringence in single crystals [28].

$\mathrm{NaNbO}_{3}$ powder exhibits a crystallographic particle size effect, with micron, submicron and nano sized powders of $\mathrm{NaNbO}_{3}$ exhibiting $\mathrm{Pbcm}, \mathrm{Pmc}_{1}$ and $\mathrm{Pmma}$ structures, respectively $[29,30]$. While this is an interesting phenomenon, its technological relevance is limited unless these structures can be stabilized in ceramics, in which case they would be expected to exhibit different polarization behavior. Obtaining dense ceramics with small grain sizes through conventional sintering is technically challenging, due to the significant grain growth that occurs at high temperatures. Recently, ferroelectric behaviour has been reported in nano-grain sized $\mathrm{NaNbO}_{3}$ ceramics [31], which is inconsistent with the non-polar structure observed in nano sized powders [29]. Moreover, it has been reported that depending on processing conditions large grain sized ceramics may exhibit the polar strucure [32]. In order to clarify the physical mechanism of the size effect in $\mathrm{NaNbO}_{3}$ ceramics, in this work, spark plasma sintering (SPS) is used to prepare ceramics of $\mathrm{NaNbO}_{3}$ with sub-micron and nano- sized grains, which are compared to micron grain-sized ceramics prepared by conventional sintering. The phase and polarization behaviors in these ceramics are examined and reveal new evidence about the nature of the Pnmm to Pbma phase transition. The transition between these AFE phases appears to be indirect, involving the $P 2{ }_{1}$ ma FE phase as an intermediate, which can be stabilized through grain size control. Both structure and polarization behavior are shown to be grain size dependent.

\section{Experimental}

$\mathrm{NaNbO}_{3}$ samples with micron and sub-micron sized grains were first prepared through solid state synthesis and then sintered in either a conventional box furnace or using SPS. Stoichiometric amounts of the starting materials $\mathrm{Na}_{2} \mathrm{CO}_{3}$ (Alfa Aesar, 99.5\%, pre-heated at $200{ }^{\circ} \mathrm{C}$ for $24 \mathrm{~h}$ ) and $\mathrm{Nb}_{2} \mathrm{O}_{5}$ (Alfa Aesar, 99.9\%) were ground in ethanol in a nylon container, with zirconia balls, using a planetary ball mill (QM-3SP4, Nanjing University Instrument Plant, China) for $4 \mathrm{~h}$ at $360 \mathrm{rpm}$. The resulting slurry was dried and sieved $(250 \mu \mathrm{m})$. The powder was then calcined at $1000{ }^{\circ} \mathrm{C}$ for $2 \mathrm{~h}$ in an alumina crucible. After calcination, the powders were ball milled again for $4 \mathrm{~h}$ at 360 rpm in ethanol, dried and sieved. The powder was then pressed uniaxially into pellets 
of $10 \mathrm{~mm}$ diameter and ca. $1 \mathrm{~mm}$ thickness at a pressure of $150 \mathrm{MPa}$. Pellets were sintered at $1200{ }^{\circ} \mathrm{C}$ for $3 \mathrm{~h}$ and then slow cooled to form micron grain sized $\mathrm{NaNbO}_{3}$. Sub-micron grain sized $\mathrm{NaNbO}_{3}$ was prepared by SPS (HPD 25/1, FCT, Rauenstein, Germany) at $950^{\circ} \mathrm{C}$ for 5 min at a uniaxial pressure of $60 \mathrm{MPa}$. Samples were pressed in a $20 \mathrm{~mm}$ diameter graphite die surrounded by carbon foil and pressing carried out under vacuum. Samples of nano-grain sized $\mathrm{NaNbO}_{3}$ were synthesized via a sol-gel method. Ammonium niobate oxalate hydrate $\left(\mathrm{C}_{4} \mathrm{H}_{4} \mathrm{NNbO}_{9} \cdot x \mathrm{H}_{2} \mathrm{O}, 18.5 \mathrm{~g}\right.$, Aldrich, 99.99\%) and $\mathrm{NaNO}_{3}$ (5.2 g, Hopkin \& Williams, 99\%) were separately dissolved in 5 $\mathrm{ml}$ of distilled water. Gelatine (15 g, Sigma-Aldrich, from bovine skin) was dissolved in $100 \mathrm{ml}$ of distilled water, while stirring at $60^{\circ} \mathrm{C}$ in an oil bath. After the clear solution was formed, the sodium nitrate and ammonium niobate oxalate solutions were added and the mixture stirred continuously at $80^{\circ} \mathrm{C}$. After approximately $4 \mathrm{~h}$, a viscous gel was formed, which was transferred to an alumina crucible and heated at $600{ }^{\circ} \mathrm{C}$ for 12 h. The resulting powder was then sintered at $850^{\circ} \mathrm{C}$ for $5 \mathrm{~min}$ at a pressure of $60 \mathrm{MPa}$ using SPS. SPS processed samples were subsquently annealed at 600 and $800{ }^{\circ} \mathrm{C}$ for $20 \mathrm{~h}$, for nano- and sub-micron sized ceramics, respectively, to remove residual carbon arising from the carbon foil. Pellet densities were measured by the Archimedes method.

The morphology of gold coated fractured cross-sections of pelletized samples was examined using scanning electron microscopy (FEI Inspect F SEM), with an incident beam generated at $20 \mathrm{kV}$. Energy-dispersive X-ray spectroscopy (EDS) was used to examine the elemental ratio in the carbon coated ceramics. For X-ray powder diffraction and thermal analysis, pellets were crushed and ground into fine powders. XRD data were collected on a PANalytical X'Pert Pro diffractometer, fitted with an

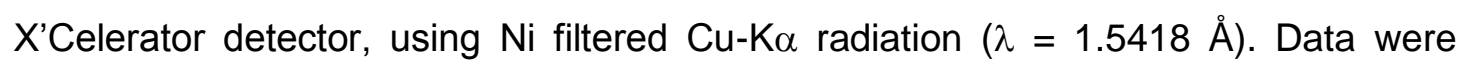
collected at room temperature in flat plate $\theta / \theta$ geometry over the $2 \theta$ range $5-120^{\circ}$, with a step width of $0.0334^{\circ}$ and an effective count time $50 \mathrm{~s}$ per step. Data were modeled by Rietveld analysis using the GSAS suite of programmes [33]. Structure refinement was carried out in the orthorhombic space groups $P b m a$ and $P 2_{1}$ ma using the models of Xu et al. [34] and Johnston et al. [25], respectively. To examine the possibility of $\mathrm{Na}_{2} \mathrm{O}$ loss during synthesis, free refinement of the Na site occupancy in the Rietveld analysis was carried out. In all cases the $\mathrm{Na}$ site occupancy refined to 1.0 within estimated satndard deviation and in the final refinements these were fixed at the stoichiometric value. Differential scanning calorimetry (DSC 25. TA instruments, Asse, Belgium) was used to measure the thermal properties of the studied compositions. The 
powder was heated from $20^{\circ} \mathrm{C}$ to $400^{\circ} \mathrm{C}$ at a heating rate of $2{ }^{\circ} \mathrm{C} \mathrm{min}-1$, held for 5 min. and then cooled to $20^{\circ} \mathrm{C}$ at a cooling rate of $2{ }^{\circ} \mathrm{C} \mathrm{min}-1$.

For electrical measurements, pellets were ground and polished into blocks of approximate dimensions $3 \mathrm{~mm} \times 3 \mathrm{~mm} \times 0.25 \mathrm{~mm}$ and the two largest surfaces coated with silver paste (Gwent Electronic Materials Ltd. Pontypool, U.K.). The samples were heated to $300{ }^{\circ} \mathrm{C}$ to decompose the paste. The dielectric permittivity and loss were measured as a function of temperature using an Agilent 4284A LCR meter, over the temperature range $25{ }^{\circ} \mathrm{C}$ to $550{ }^{\circ} \mathrm{C}$. I-E and P-E loops were measured at room temperature using a ferroelectric hysteresis measurement tester (NPL, UK). The electric voltage was applied in a triangular waveform [35]. A quasi-static ZJ-3B $d_{33}$ meter (Institute of Acoustics Academia Sinica, China) was used to measure the piezoelectric coefficient $\left(d_{33}\right)$ of samples.

\section{Results and Discussion}

The morphology of the studied ceramics is shown in Fig. S1. The average grain sizes were ca. $300 \mathrm{~nm}, 750 \mathrm{~nm}$ and $1.5 \mu \mathrm{m}$ for nano-, sub-micron and micron sized $\mathrm{NaNbO}_{3}$ ceramics, respectively. For the nano-sized sample, smaller (ca. $50 \mathrm{~nm}$ ) subgrains are seen on the surface of the main grains (Fig. S1a), as seen in previous studies [36,37]. The densities of the nano-, sub-micron and micron grain sized ceramics were found to be $4.440 \mathrm{~g} \mathrm{~cm}^{-1}, 4.515 \mathrm{~g} \mathrm{~cm}^{-1}$ and $4.342 \mathrm{~g} \mathrm{~cm}^{-1}$, respectively, corresponding to $97 \%, 99 \%$ and $95 \%$ of the respective theoretical densities. EDS analysis (Fig. S2 and Table S1) confirms the expected stocihiometry in all samples, with a constant $\mathrm{Na} / \mathrm{Nb}$ ratio and no evidence of signifcant oxide ion vacancy concentration. The results are consistent with minimal volatilization of $\mathrm{Na}_{2} \mathrm{O}$ and minimal reduction of $\mathrm{Nb}^{5+}$.

The X-ray powder diffraction (XRD) data for the crushed pellets were fitted using two models, the non-polar Pbma model and the polar $P 2{ }_{1}$ ma model. The fitted diffraction profiles for these two models are presented in Fig. S3 and Fig. S4, respectively, with the results summarized in Table $S 1$. Details of the fits to the diffraction profiles are shown in Fig. 1. Bragg peaks corresponding to (221) and (122) reflections in Pbma or (211) and (112) reflections in $P 2_{1}$ ma between $38^{\circ}$ and $39^{\circ} 2 \theta$ were well fitted regardless of the space group. The $P 2{ }_{1}$ ma space group is a sub-group of Pbma in which the $b$-axis length is halved and therefore these reflections appear in both space groups. In contrast, clear differences are seen in the patterns between $36^{\circ}$ and $37^{\circ} 2 \theta$. The (230) peak at ca. $36.9^{\circ} 2 \theta$ is unique to the Pbma supercell and allows 
for a clear distinction between these two structures. As indicated by the R-factors, Fig. 1 shows that the Pbma model better fits the data for micron grain sized $\mathrm{NaNbO}_{3}$, while the $P 2{ }_{1}$ ma model better fits the data for nano-grain sized $\mathrm{NaNbO}_{3}$. In the case of the sub-micron grain sized sample, while comparison of R-factors revealed no preference in the space group, the fit in the region shown in Fig. 1 for the two models suggests a mixture of the two phases. This may be interpreted as traces of the polar phase in a matrix of the non-polar phase.

The differential scanning calorimetry (DSC) thermograms for the three studied samples are shown in Fig. S5. Endothermic peaks were observed on heating at ca. $315^{\circ} \mathrm{C}, 325^{\circ} \mathrm{C}$ and $360{ }^{\circ} \mathrm{C}$ for nano-, sub-micron and micron grain sized $\mathrm{NaNbO}_{3}$ samples, respectively. The temperatures of the corresponding exothermic peaks are lower than those of the endothermic peaks and are associated with a first order phase transition [17]. This transition occurs at a higher temperature for the larger grain sized $\mathrm{NaNbO}_{3}$ sample. This indicates that there is a lower polarization in the smaller grain sized ceramics, which leads to the lower phase transition temperatures. For micron grain-sized $\mathrm{NaNbO}_{3}$, a step in the DSC thermogram is observed at ca. $85{ }^{\circ} \mathrm{C}$ on heating, with a corresponding change in slope at the same temperature during cooling. Although no similar steps or changes in slope are seen on heating in the thermograms of nano- and sub-micron grain sized samples, there is a small change in slope on cooling at this temperature for these samples, but less pronounced than in the micron grain sized sample. Such features are typically associated with second order transitions.

Dielectric permittivity $\left(\varepsilon^{\prime}\right)$ and loss (tan $\left.\delta\right)$ were measured at selected frequencies on cooling from $600{ }^{\circ} \mathrm{C}$ to avoid moisture effects (Fig. 2). Dielectric loss was found to be more significant in the nano- and sub-micron grain sized ceramcs. This can be attributed to a small degree of reduction in the ceramics during SPS processing, which introduces low levels of oxide ion vacancies. These remain, despite subsequent annealing of the sintered ceramics, but the levels are lower than the detaction limit of EDS. A clear peak in dielectric permittivity at ca. $300{ }^{\circ} \mathrm{C}$ is observed for all samples. This peak has been attributed to the phase transition from the AFE R-phase, in space group Pnmm above $300{ }^{\circ} \mathrm{C}$, to the AFE P-phase, in space group Pbma [17]. The fact that the Pbma phase is not observed in the nano-grain sized sample, but the dielectric permittivity peak at $300^{\circ} \mathrm{C}$ is still seen, suggests that this transition is more complex. It is also noteworthy that this peak broadens with decreasing grain size, indicating that 
the phase transition occurs over an increasing temperature range, consistent with a decrease in crystallographic order.

The results suggest that the reported $P n m m \rightarrow P b m a$ phase transition on cooling actually occurs via the $P 2_{1}$ ma phase, i.e.

$$
\mathrm{Pnmm} \rightarrow \mathrm{P2}{ }_{1} \mathrm{ma} \rightarrow \mathrm{Pbma}
$$

The reduction in grain size has the effect of suppressing the second phase transition. Thus, in the nano-grain sized sample the polar $P 2{ }_{1}$ ma phase is observed at room temperature [31] and the $P n m m \rightarrow P 2_{1}$ ma transition occurs over a wide temperature range, leading to a broadening of the dielectric permittivity peak. This type of phase behavior has been noted before in $\mathrm{BaTiO}_{3}$, where a reduction of grain size leads to suppression of the cubic to tetragonal phase transition on cooling [6]. The Pnmm to $P{ }_{1}$ ma phase transition involves a tilting of the $\mathrm{NbO}_{6}$ octahedra and a change in the tilting system from $a^{-} b^{+} a^{+}$to $a^{-} b^{+} a$, while the $P 2{ }_{1}$ ma to $P b m a$ transition involves a doubling of the unit cell along the $b$-axis, with the layers in the top half and bottom half having opposing tilt directions (Fig. 3).

Inspection of the dielectric permittivity plot for micron grain size $\mathrm{NaNbO}_{3}$ from 150 ${ }^{\circ} \mathrm{C}$ to $100{ }^{\circ} \mathrm{C}$ (Fig. 2c), reveals an anomaly not present in the plots for the other samples. This anomaly has also been found by other researchers, but its origin is still under debate [38]. Raman spectroscopic and synchrotron X-ray diffraction studies concluded that the so called P-phase was actually three different phases, each with a different stability range, i.e. a monoclinic phase from $23{ }^{\circ} \mathrm{C}$ to $137{ }^{\circ} \mathrm{C}$, an incommensurate phase (INC) from $137^{\circ} \mathrm{C}$ to $187^{\circ} \mathrm{C}$ and an orthorhombic phase from $187^{\circ} \mathrm{C}$ to $360^{\circ} \mathrm{C}$ [23]. An in situ TEM study confirmed the existence of the INC phase in $\mathrm{NaNbO}_{3}$ at $165^{\circ} \mathrm{C}$ [39]. However, Koruza et al. reported that the observed dielectric anomaly in $\mathrm{NaNbO}_{3}$ at around $150{ }^{\circ} \mathrm{C}$ was associated with relaxor behavior and was caused by ferroelectric nano-regions embedded in an antiferroelectric matrix, rather than a discrete phase transition [40]. Indeed, later neutron diffraction studies by Mishra et al. failed to identify any phase transitions in the P-phase region [24].

The present results indicate that the $P 2{ }_{1} m a$ phase is an intermediate phase between the $\mathrm{R}(\mathrm{Pmnm})$ and $\mathrm{P}$ (Pbma) phases. Therefore, it is possible that the anomaly seen in micron grain size $\mathrm{NaNbO}_{3}$ from $150{ }^{\circ} \mathrm{C}$ to $100{ }^{\circ} \mathrm{C}$ (Fig. 2c) is associated with a phase transition from the $P 2{ }_{1}$ ma phase to the Pbma phase. In order to investigate this anomaly, dielectric permittivity measurements were carried out on a poled sample of micron grain sized $\mathrm{NaNbO}_{3}$ on heating and cooling (Fig. 4). The peak 
associated with the anomaly was found to be absent on heating, but present on cooling. This may be explained by considering the effect of poling on the sample. The high field used in poling the sample likely induces the transition from the Pbma to the $P 2{ }_{1}$ ma phase at room temperature, so that on heating if indeed the anomaly is associated with the $P 2{ }_{1}$ ma to $P b m a$ transition, it would be expected to be absent. On heating the poled sample above $300{ }^{\circ} \mathrm{C}$ to the R-phase region, depoling occurs and on subsequent cooling the anomaly reappears. In contrast, with smaller grain size $\mathrm{NaNbO}_{3}$ samples, the anomaly is always absent both on heating and cooling. This suggests that the Pbma phase is only a minor phase or not formed at all in small grain size $\mathrm{NaNbO}_{3}$ samples.

On cooling, $\mathrm{NaNbO}_{3}$ samples from $300{ }^{\circ} \mathrm{C}$, they undergo the $\mathrm{Pnmm} \rightarrow P 2_{1}$ ma phase transition, i.e. from a non-polar to a polar structure. This transition involves the growth of polar regions. The polar region surface would be charged as a result of this polarization and the resulting field is known as the depolarizing field, $E_{\mathrm{d}}[5]$. $E_{\mathrm{d}}$ therefore represents a positive contribution to the free energy of the $P 2{ }_{1}$ ma phase. For nanograin sized $\mathrm{NaNbO}_{3}$, the size of the polar regions is constrained by the small grain size and consequently for a single polar region $E_{d}$ is small. This means, that in small grain sized samples, the $P 2_{1}$ ma phase has a lower free energy than the Pbma phase and there is no significant energy to be gained in ordering to form the Pbma phase. Hence the $P 2{ }_{1}$ ma phase is stabilized at room temperature. On the other hand, in large grain sized samples, the growth of the polar region at the $P n m m \rightarrow P 2_{1}$ ma phase transition is no longer limited by the grain size and polar regions continue to grow to give larger regions, with larger $E_{\mathrm{d}}$. This makes the $P{ }_{1}$ ma phase less stable with respect to the Pbma phase. Minimization of $E_{d}$ typically involves polar regions splitting into neighboring regions with antiparallel polarization [41]. This process involves the ordering of adjacent dipoles and eventually leads to the formation of the AFE Pbma phase. Thus in large grain sized samples, the ordering process is energetically favored as the sample is cooled and the $P 2{ }_{1} m a \rightarrow P b m a$ phase transition is observed.

Low-field polarization-electric field (P-E) and current-electric field (I-E) loops for the studied samples are shown in Fig. 5. All samples showed tilted I-E loops associated with a leakage current. Only micron grain sized $\mathrm{NaNbO}_{3}$ shows current peaks at around $\pm 1 \mathrm{kV} \mathrm{mm}^{-1}$. These might be associated with a field induced phase transition e.g. from the $P b m a$ phase to the $P 2{ }_{1}$ ma phase or with a coercive field, $E_{c}$ arising from some residual $P 2{ }_{1}$ ma phase within the predominant $P b m a$ matrix. For smaller grain sized samples, where the polar phase was evident in X-ray diffraction data, no current peaks are seen at these fields, which suggests that the applied electric field was 
insufficient to facilitate domain switching. The measured, $d_{33}$ value of the micron grain sized sample post experiment was $0.6 \pm 0.1 \mathrm{pC} \mathrm{N}^{-1}$, confirming a small remnant polarization after application of an applied field of $6 \mathrm{kV} \mathrm{mm}^{-1}$. Those for the nano- and sub-micron grain sized samples were 15.4 and $2.2 \mathrm{pC} \mathrm{N}^{-1}$, respectively.

$\mathrm{P}-\mathrm{E} / \mathrm{I}-\mathrm{E}$ loops measured under high electric fields are shown in Fig. 6. Two current peaks are observed in the I-E loops for the sub-micron and nano-grain sized samples and can be attributed to domain switching of the FE phase. It is noticeable that the coercive field of the sub-micron grain sized sample is lower than that of the nano-grain sized sample (ca. $6 \mathrm{kV} \mathrm{mm}^{-1}$ and ca. $8 \mathrm{kV} \mathrm{mm}^{-1}$, respectively). The higher grain boundary concentration in nano-grain sized $\mathrm{NaNbO}_{3}$ increases the so called "pinning effect" i.e. they serve as barriers to domain switching and consequently a high coercive field is required for domain switching to occur [42]. Four current peaks are observed in the I-E loop of the micron grain sized sample (Fig. 6c). The lower field peak at ca. 4.5 $\mathrm{kV} \mathrm{mm}{ }^{-1}$ is attributed to the transition from the FE $P 2{ }_{1}$ ma phase to the AFE Pbma phase, while the second peak at ca. $7 \mathrm{kV} \mathrm{mm}^{-1}$ is associated with the reverse transition. The values of the applied fields at these transitions are denoted $E_{\mathrm{AFE}}$ and $E_{\mathrm{FE}}$, respectively. Thus at points 1 and 3 in Fig. $6 \mathrm{c}$ the sample is in the FE phase, while at point 2, it is in the AFE state. This field induced AFE phase was also observed in $\mathrm{NaNbO}_{3}$ based ceramics and confirmed by XRD measurements [43].

Fig. 7 shows fitted X-ray diffraction patterns of virgin and poled (at $14 \mathrm{kV} \mathrm{mm}^{-1}$ ) micron sized $\mathrm{NaNbO}_{3}$ ceramics, modeled using the $\mathrm{Pbma}$ and $\mathrm{P}_{2}{ }_{1}$ ma structures, with those for sub-micron and nano-grain sized ceramics given in Figs. S6 and S7, respectively. There is a clear difference observed in the $2 \theta$ range $35-39^{\circ}$ between the virgin and poled samples in the micron grain sized ceramic. For this sample, the diffraction pattern of virgin $\mathrm{NaNbO}_{3}$ ceramic is clearly better fitted using the non-polar Pbma structure, while after poling both models fit equally well and cannot be distinguished. However, the pattern of the poled sample in this $2 \theta$ range closely resembles that of the nano-grain sized powder sample shown in Fig. 1, which was dominated by the polar phase. This suggests that high electric field induces a phase transition from non-polar to polar structures in micron grain sized $\mathrm{NaNbO}_{3}$ ceramics. Similar behaviour is seen in the sub-micron grain sized ceramic (Fig. S6), while in the nano-grain sized ceramic the polar structure appears to be maintained throughout.

In order to further investigate the anomaly in the dielectric permittivity seen in micron grain sized $\mathrm{NaNbO}_{3}$, variable temperature P-E/l-E measurements were performed at low electric fields (Fig. S8). With increasing temperature, the current peaks shift to lower electric field. As discussed above, these current peaks are attributed to domain 
switching in local FE regions in a non-polar matrix. In addition, the magnitude of the current peaks decreases with increasing temperature and the peaks disappear at 150 ${ }^{\circ} \mathrm{C}$. This suggests that the extent of the polar regions diminishes with increasing temperature, leaving only the non-polar AFE matrix, i.e. the pure Pbma phase. Indeed, further increase in temperature up to $200{ }^{\circ} \mathrm{C}$ leads to a normal FE hysteresis loop at high field, with only 2 current peaks in the I-E loop (Fig. 8), compared to the 4 current peaks seen at room temperature (Fig. 6c). The results are consistent with the dielectric anomaly seen at $\mathrm{ca} .150{ }^{\circ} \mathrm{C}$ as being due to the $\mathrm{Pbma} \leftrightarrow P 2{ }_{1}$ ma transition.

\section{Conclusions}

Sodium niobate ceramics with different grain sizes (300 nm to $1.4 \mu \mathrm{m}$ ) have been successfully prepared using solid state and sol-gel methods, with conventional sintering and SPS. For micron grain sized ceramics, samples were predominantly in the AFE P-phase (space group Pbma), while nano-grain sized samples exhibited a structure with $P 2{ }_{1}$ ma symmetry that showed FE behaviour. Sub-micron grain sized samples exhibited a mixture of both phases. An anomaly seen in the dielectric permittivity of micron grain-sized $\mathrm{NaNbO}_{3}$ is attributed to the transition between AFE and FE phases. The transition from FE to AFE phases involves ordering of dipoles to generate an ordered structure with opposing tilt directions, resulting in doubling of the unit cell. The results of this work suggest that the FE $P 2_{1}$ ma phase is always formed directly from the R-phase at $300^{\circ} \mathrm{C}$ on cooling and that the previously reported $\mathrm{R} \leftrightarrow \mathrm{P}$ phase transition does not occur directly, but proceeds via the $P 2{ }_{1}$ ma phase, with the $P 2{ }_{1}$ ma to Pbma (FE to AFE) transition occurring at around $150{ }^{\circ} \mathrm{C}$. The work has shown that the growth of FE domains in small grain sized ceramics is suppressed, making the FE to AFE transition less energetically favourable, allowing for the FE $P 2_{1}$ ma phase to be stabilized to room temperature. The results indicate that careful control of microstructure through processing conditions can allow for tailoring of dielectric behaviour in ceramics, allowing for its use in the design and manufacture of a variety of new electronic devices.

\section{Acknowledgements}

The authors are grateful to the Materials Research Institute at Queen Mary for studentship funding for Hangfeng Zhang. The authors are also grateful for financial support from EPSRC (MASSIVE project EP/L017695/1; $\quad$ Teranet fund EP/M00306X/1). 


\section{References}

[1] X. Liu, X. Tan, Giant Strains in Non-Textured (Bi1/2Na1/2)TiO3-Based LeadFree Ceramics, Adv. Mater. 28 (2016) 574-578.

[2] J. Li, F. Li, Z. Xu, S. Zhang, Multilayer Lead-Free Ceramic Capacitors with Ultrahigh Energy Density and Efficiency, Adv. Mater. 30 (2018) 1802155.

[3] P. Li, J. Zhai, B. Shen, S. Zhang, X. Li, F. Zhu, X. Zhang, Ultrahigh Piezoelectric Properties in Textured (K,Na)NbO 3 -Based Lead-Free Ceramics, Adv. Mater. 30 (2018) 1705171.

[4] A.M. Manjón-Sanz, M.R. Dolgos, Applications of Piezoelectrics: Old and New, Chem. Mater. 30 (2018) 8718-8726.

[5] D. Damjanovic, Ferroelectric, dielectric and piezoelectric properties of ferroelectric thin films and ceramics, Rep. Prog. Phys. 61 (1998) 1267-1324.

[6] Z. Zhao, V. Buscaglia, M. Viviani, M.T. Buscaglia, L. Mitoseriu, A. Testino, M. Nygren, M. Johnsson, P. Nanni, Grain-size effects on the ferroelectric behavior of dense nanocrystalline BaTiO3 ceramics, Phys. Rev. B. 70 (2004) 241071241078.

[7] H.T. Martirena, J.C. Burfoot, Grain-size effects on properties of some ferroelectric ceramics, J. Phys. C Solid State Phys. 7 (2001) 3182-3192.

[8] T.M. Kamel, G. de With, Grain size effect on the poling of soft

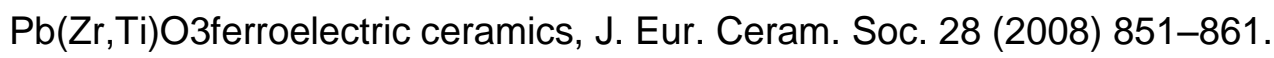

[9] T. Shet, R. Bhimireddi, K.B.R. Varma, Grain size-dependent dielectric, piezoelectric and ferroelectric properties of Sr2Bi4Ti5O18 ceramics, J. Mater. Sci. 51 (2016) 9253-9266.

[10] Z. Yang, H. Du, S. Qu, Y. Hou, H. Ma, J. Wang, J. Wang, X. Wei, Z. Xu, Significantly enhanced recoverable energy storage density in potassiumsodium niobate-based lead free ceramics, J. Mater. Chem. A. 4 (2016) 1377813785.

[11] T. Shao, H. Du, H. Ma, S. Qu, J. Wang, J. Wang, X. Wei, Z. Xu, Potassiumsodium niobate based lead-free ceramics: novel electrical energy storage materials, J. Mater. Chem. A. 5 (2017) 554-563.

[12] M. Zhou, R. Liang, Z. Zhou, S. Yan, X. Dong, Novel Sodium Niobate-Based Lead-Free Ceramics as New Environment-Friendly Energy Storage Materials with High Energy Density, High Power Density, and Excellent Stability, ACS Sustain. Chem. Eng. 6 (2018) 12755-12765.

[13] H. Shimizu, H. Guo, S.E. Reyes-Lillo, Y. Mizuno, K.M. Rabe, C.A. Randall, Lead-free antiferroelectric: xCaZrO $3-(1-x) \mathrm{NaNbO} 3$ system $(0 \leq x \leq 0.10)$, Dalt. Trans. 44 (2015) 10763-10772. 
[14] E. Cross, Lead-free at last, Nature. 432 (2004) 666-668.

[15] Y. Saito, H. Takao, T. Tani, T. Nonoyama, K. Takatori, T. Homma, T. Nagaya, M. Nakamura, "Lead-free piezoceramics," Nature. 432 (2004) 84-87.

[16] F. Bortolani, A. Del Campo, J.F. Fernandez, F. Clemens, F. Rubio-Marcos, High strain in $(\mathrm{K}, \mathrm{Na}) \mathrm{NbO}$-based lead-free piezoelectric fibers, Chem. Mater. 26 (2014) 3838-3848.

[17] H.D. Megaw, The seven phases of sodium niobate, Ferroelectrics. 7 (1974) 87-89.

[18] T.F.W. Barth, Die Kristallstruktur von Perowskit und verwandten Verbidungen, Nor. Geol Tidsskr. 8 (1925) 201-216.

[19] P. Vousden, The structure of ferroelectric sodium niobate at room temperature, Acta Crystallogr. 4 (1951) 545-551.

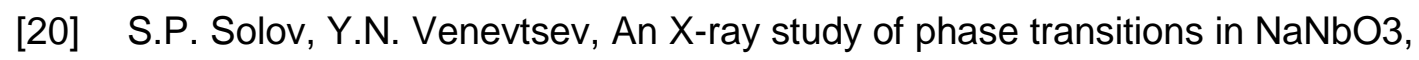
Sov. Physics-Crystallography. 6 (1961) 171-175.

[21] A.C. Sakowski-Cowley, K. Lukaszewicz, H.D. Megaw, K. Łukaszewicz, H.D. Megaw, The structure of sodium niobate at room temperature, and the problem of reliability in pseudosymmetrie structures, Acta Cryst. B. 25 (1969) 851-865.

[22] C.N.W. Darlington, K.S. Knight, On the lattice parameters of sodium niobate at room temperature and above, Phys. B Condens. Matter. 266 (1999) 368-372.

[23] Y.I. Yuzyuk, P. Simon, E. Gagarina, L. Hennet, D. Thiaudière, V.I. Torgashev, S.I. Raevskaya, I.P. Raevskii, L.A. Reznitchenko, J.L. Sauvajol, Modulated phases in $\mathrm{NaNbO} 3$ : Raman scattering, synchrotron x-ray diffraction, and dielectric investigations, J. Phys. Condens. Matter. 17 (2005) 4977-4990.

[24] S.K. Mishra, R. Mittal, V.Y. Pomjakushin, S.L. Chaplot, Phase stability and structural temperature dependence in sodium niobate: A high-resolution powder neutron diffraction study, Phys. Rev. B - Condens. Matter Mater. Phys. 83 (2011) 1341051-1341059.

[25] K.E. Johnston, C.C. Tang, J.E. Parker, K.S. Knight, P. Lightfoot, S.E. Ashbrook, The polar phase of $\mathrm{NaNbO3:} \mathrm{A} \mathrm{combined} \mathrm{study} \mathrm{by} \mathrm{powder}$ diffraction, solid-state NMR, and first-principles calculations, J. Am. Chem. Soc. 132 (2010) 8732-8746.

[26] L.E. Cross, B.J. Nicholson, LV. The optical and electrical properties of single crystals of sodium niobate, London, Edinburgh, Dublin Philos. Mag. J. Sci. 46 (1955) 453-466.

[27] V.A. Shuvaeva, R.S. V Lindeman, E. Fesenko, V.G. Smotrakov, R. Academy, R.S. V Lindeman, V.G. Smotrakov, crystal structure of the electric-field - 
induced ferroelectric phase of NaNbO3, Ferroelectrics. 141 (1993) 307-311.

[28] A. V Ulinzheyev, O.E. Fesenko, V.G. Smotrakov, Super-high field-induced phase transitions in NaNbO 3 crystals, Ferroelectr. Lett. Sect. 12 (1990) 1721.

[29] Y. Shiratori, A. Magrez, J. Dornseiffer, F. Haegel, C. Pithan, R. Waser, Polymorphism in Micro-, Submicro-, and Nanocrystalline NaNbO 3, J. Phys. Chem. B. 109 (2005) 20122-20130.

[30] S. Ji, H. Liu, Y. Sang, W. Liu, G. Yu, Y. Leng, Synthesis, structure, and piezoelectric properties of ferroelectric and antiferroelectric $\mathrm{NaNbO} 3$ nanostructures †, (2014) 7598-7604.

[31] A. Phys, Macroscopic ferroelectricity and piezoelectricity in nanostructured NaNbO3 ceramics, 122901 (2017).

[32] H. Shimizu, K. Kobayashi, Y. Mizuno, C.A. Randall, Advantages of low partial pressure of oxygen processing of alkali niobate: $\mathrm{NaNbO3}$, J. Am. Ceram. Soc. 97 (2014) 1791-1796.

[33] A.C. Larson, R.B. Dreele, Los Alamos National Laboratory Report, 1987.

[34] H. Xu, Y. Su, M. Lou Balmer, A. Navrotsky, A new series of oxygen-deficient perovskites in the NaTixNb1-xO3-0.5x system: Synthesis, crystal chemistry, and energetics, Chem. Mater. 15 (2003) 1872-1878.

[35] G. Viola, T. Saunders, X. Wei, K.B. Chong, H. Luo, M.J. Reece, H. Yan, Contribution of piezoelectric effect, electrostriction and ferroelectric/ferroelastic switching to strain-electric field response of dielectrics, J. Adv. Dielectr. 03 (2013) 1350007.

[36] Z. Shen, D. Grüner, M. Eriksson, L.M. Belova, C.W. Nan, H. Yan, Ordered coalescence of nano-crystals in alkaline niobate ceramics with high remanent polarization, J. Mater. 3 (2017) 267-272.

[37] Z. Shen, H. Yan, D. Grüner, L.M. Belova, Y. Sakamoto, J. Hu, C. Nan, T. Höche, M.J. Reece, Ferroelectric ceramics with enhanced remnant polarization by ordered coalescence of nano-crystals, J. Mater. Chem. 22 (2012) 23547.

[38] X. Tan, C. Ma, J. Frederick, S. Beckman, K.G. Webber, The antiferroelectric $\leftrightarrow$ ferroelectric phase transition in lead-containing and lead-free perovskite ceramics, J. Am. Ceram. Soc. 94 (2011) 4091-4107.

[39] H. Guo, H. Shimizu, C. a. Randall, Direct evidence of an incommensurate phase in $\mathrm{NaNbO} 3$ and its implication in $\mathrm{NaNbO} 3$-based lead-free antiferroelectrics, Appl. Phys. Lett. 107 (2015) 112904.

[40] J. Koruza, J. Tellier, B. Malič, V. Bobnar, M. Kosec, Phase transitions of 
sodium niobate powder and ceramics, prepared by solid state synthesis, J. Appl. Phys. 108 (2010) 1135091-1135099.

[41] N. Spaldin, MATERIALS SCIENCE: Fundamental Size Limits in Ferroelectricity, Science. 304 (2004) 1606-1607.

[42] Y. Tan, J. Zhang, Y. Wu, C. Wang, V. Koval, B. Shi, H. Ye, R. McKinnon, G. Viola, $\mathrm{H}$. Yan, Unfolding grain size effects in barium titanate ferroelectric ceramics, Sci. Rep. 5 (2015) 9953.

[43] Y. Xu, W. Hong, Y. Feng, X. Tan, Antiferroelectricity induced by electric field in NaNbO3-based lead-free ceramics, Appl. Phys. Lett. 104 (2014) 529031529035. 

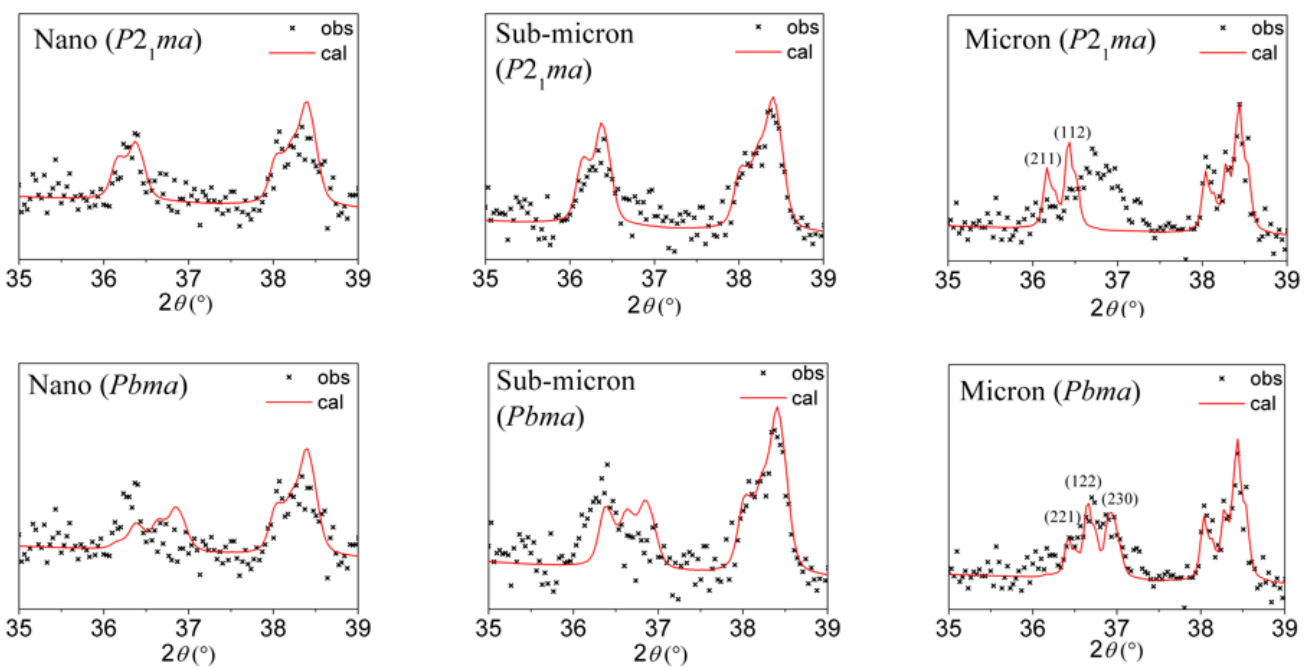

Fig. 1. Detail of the fits to X-ray diffraction profiles for $\mathrm{NaNbO}_{3}$ samples of different grain size using the $P 2{ }_{1}$ ma and $P b m a$ models. 

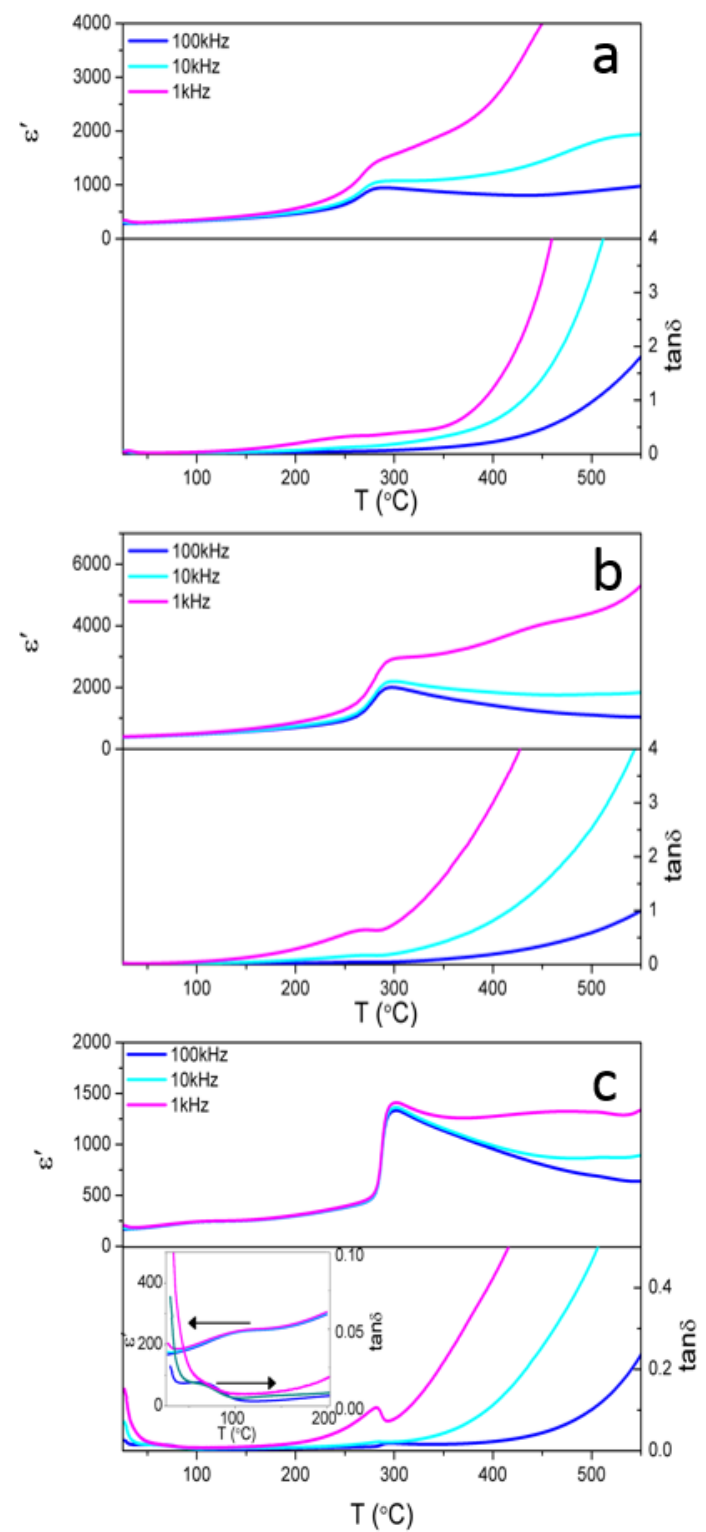

Fig. 2. Thermal variation of dielectric relative permittivity and loss on cooling for (a) nano (b) sub-micron and (c) micron grain sized $\mathrm{NaNbO}_{3}$ 

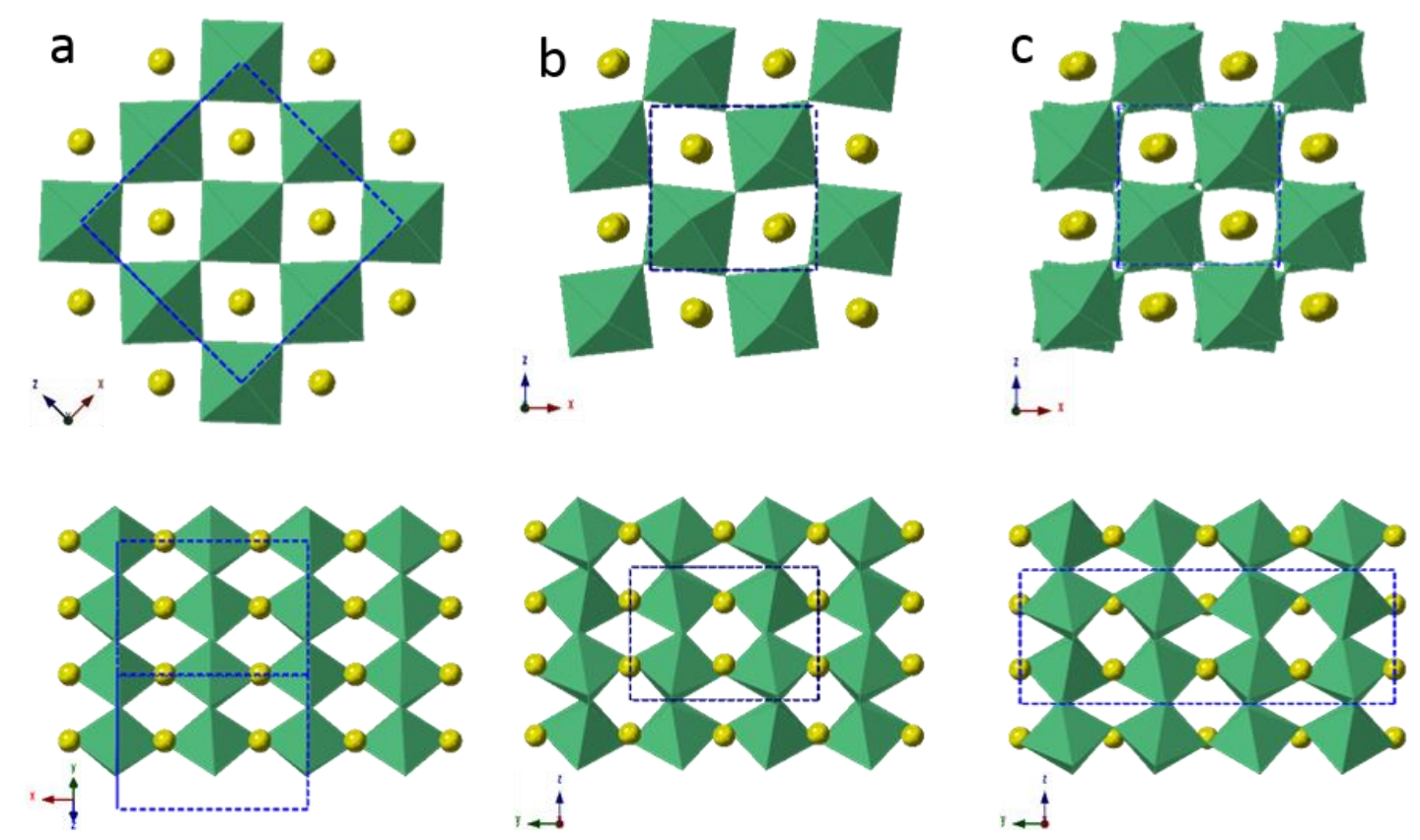

Fig. 3. Crystal structures of sodium niobate in space groups (a) $P$ nmm, (b) $P 2{ }_{1} m a$ and (c) Pbma, with the unit cells indicated by dashed lines.

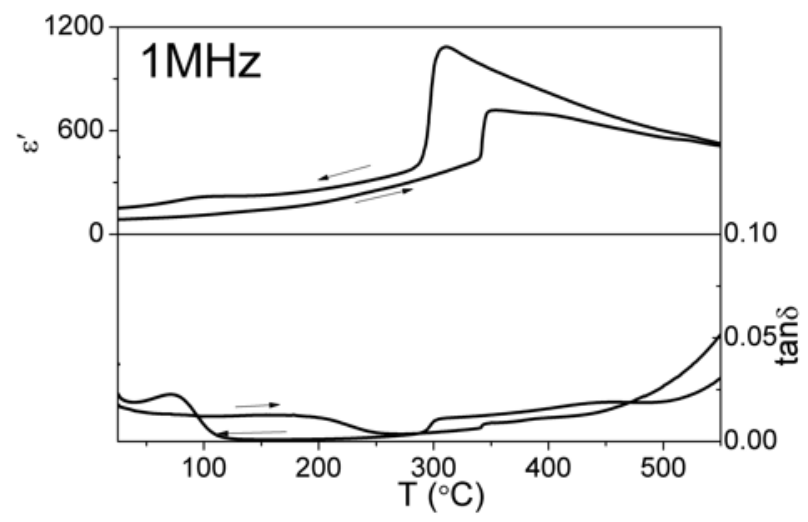

Fig. 4. Temperature dependence of dielectric permittivity and loss for poled micron grain sized $\mathrm{NaNbO}_{3}$. 

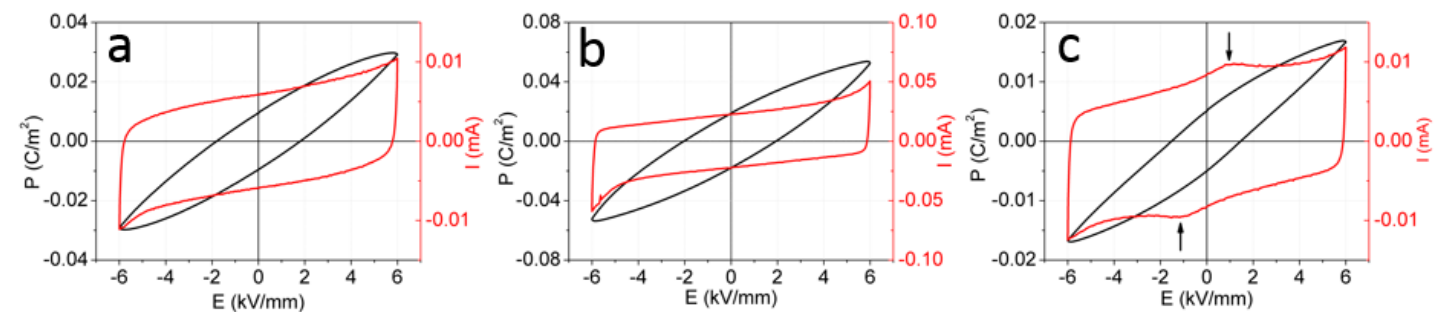

Fig. 5. P-E/l-E loops of $\mathrm{NaNbO}_{3}$ samples measured under low electric field at 10 $\mathrm{Hz}$, showing data for (a) nano-, (b) sub-micron and (c) micron grain sized samples.
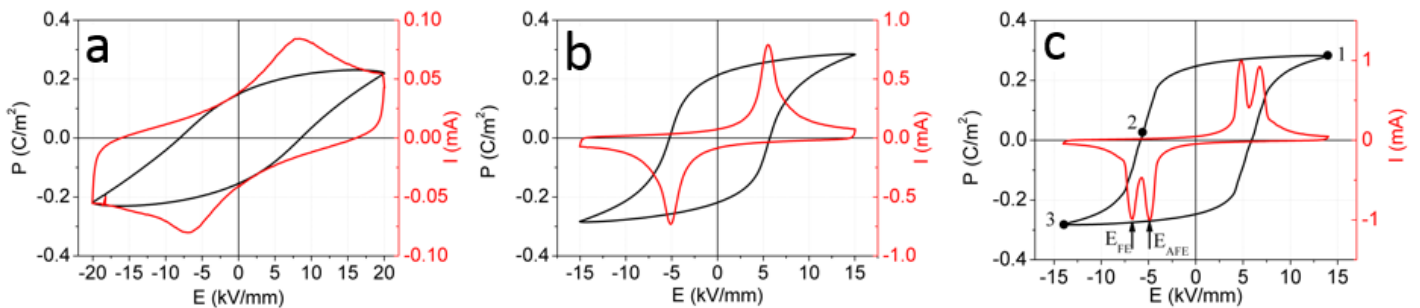

Fig. 6. P-E/I-E loops for $\mathrm{NaNbO}_{3}$ samples measured at high electric field, showing data for (a) nano, (b) sub-micron and (c) micron grain sized samples.
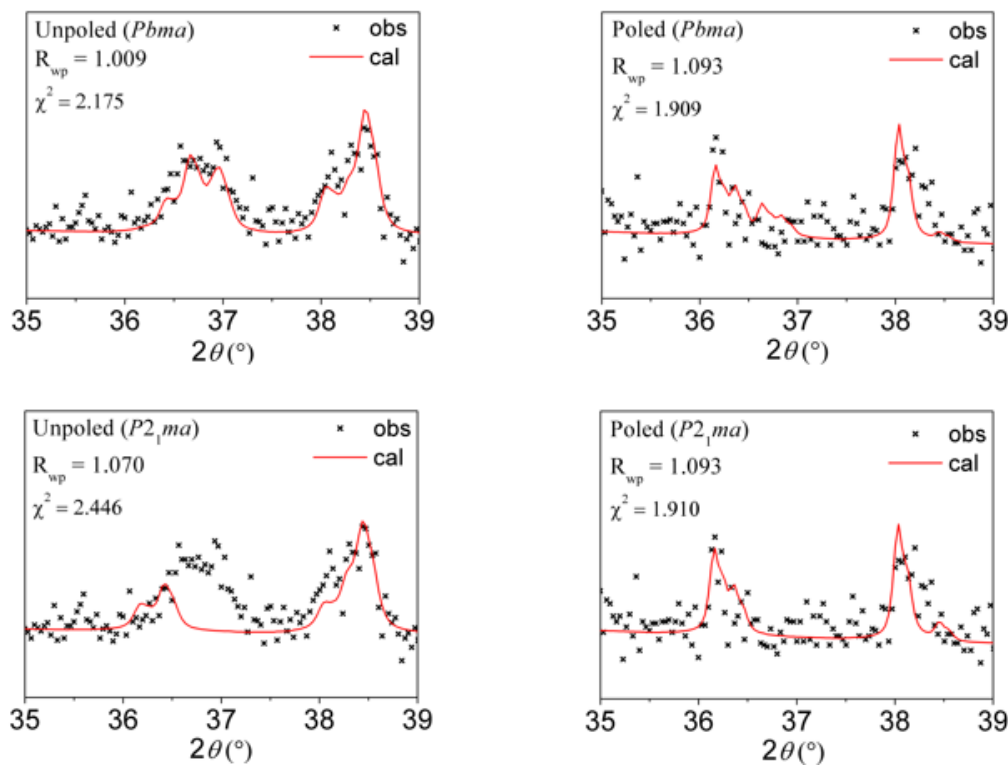

Fig. 7. Detail of fitted X-ray diffraction profiles of unpoled and poled micron sized $\mathrm{NaNbO}_{3}$ ceramics. 

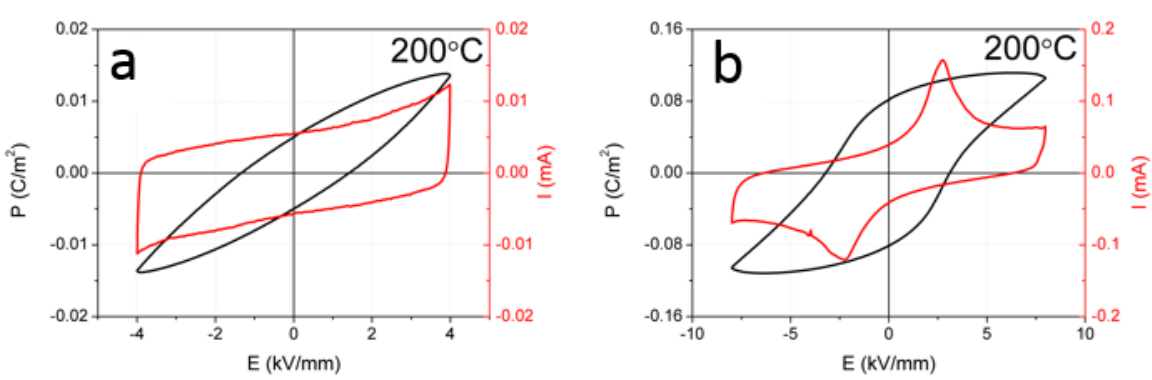

Fig. 8. I-E/P-E loops for a micron grain sized $\mathrm{NaNbO}_{3}$ ceramic measured under (a) low electric field and (b) high electric field. 